\title{
Elastodynamic contact in plate-like bodies
}

\author{
R. Sburlati \\ Department of Structural and Geotechnical Engineering, \\ Genoa University, Italy
}

\begin{abstract}
In this paper the dynamic problem of the frictionless normal low velocity impact of a rigid sphere against an elastic plate-like body is studied by assuming that the period of duration of the impact is much larger than the time employed by the elastic waves in traversing the plate after the first impact, using a static contact law taking into account the thickness of the plate and a Hertzian pressure distribution between the sphere and the plate is adopted. A non-linear second order ordinary differential equation for the dynamical value of the indentation is obtained and, by using a perturbative technique, numerical solutions concerning the contact period, the maximum contact force and the maximum indentation in terms of the plate thickness are obtained. Numerical investigations show how the time of collision decreases with the increasing of the thickness-to plate radius ratio, the maximum indentation decreases and the maximum contact force increases. All these values approach Hertz's values when the plate thickness increases. It is remarked that the solution presented here is valid only when the contact area radius is "small" in comparison to the plate thickness; if this assumption is not satisfied, the contact pressure distribution deviates significantly from the Hertzian prediction.
\end{abstract}

Keywords: contact mechanics, dynamical impact, elasticity theory, elastic plate.

\section{Introduction}

In this paper the dynamic problem of the frictionless normal low velocity impact of a rigid sphere against an elastic plate-like body is studied. In a dynamical framework an impact law taking into account the period load frequency and the thickness of the plate was obtained in [1]; nevertheless, if the period of duration of the impact is assumed much larger than the time employed by the elastic 
waves in traversing the plate after the first impact, it is possible to perform a quasi-static analysis by adopting a contact law taking into account only the thickness of the plate $[2,3]$. In such way, in section 2 the explicit form of the contact law in terms of the thickness of the plate is obtained by assuming a Hertzian pressure distribution between the sphere and the plate and in section 3 the equation of the motion is written by adopting the static contact law obtained in section 2. A non-linear second order ordinary differential equation for the dynamical value of the indentation is obtained and the use of a perturbative technique allows us to obtain the numerical values of the contact period, the maximum contact force and the maximum indentation in terms of the plate thickness.

Numerical investigation is performed in section 4 in order to evaluate the influence of the thickness-to-side in the analysis of the motion during a slow impact. It is shown how the time of collision decreases with the increasing of the thickness-to plate radius ratio; analogously, under the same assumption, the maximum indentation decreases and the maximum contact force increases. All these values approach Hertz's values when the plate thickness increases.

It is remarked that the solution presented here is valid only when the contact area radius is "small" in comparison with the plate thickness; if this assumption is not satisfied, the contact pressure distribution deviates significantly from the Hertzian prediction $[4,5]$.

\section{Contact law in plate-like bodies}

A circular plate-like body of thickness $2 h$ and radius $b$ referred to a system of cylindrical coordinates $(r, \theta, z)$ is considered such that its origin 0 is placed at the center of the middle plane.

The requirement is imposed on the plate that the transversal displacement component $w(r, z)$ is zero in $r=b$, in accordance with the notion of a simple support imposed on a thin plate. Now, if static axisymmetric loads are considered on the upper face of the circular plate, it is possible to use a Dini expansion for the radial displacement field $u(r, z)$ and a Fourier Bessel expansion for the transversal displacement $w(r, z)$ :

$$
u(r, z)=-\sum_{j=1}^{\infty} g_{j}(z) \phi_{j} J_{1}\left(\phi_{j} r\right), \quad w(r, z)=\sum_{j=1}^{\infty} f_{j}(z) J_{0}\left(\phi_{j} r\right)
$$

The functions $g_{j}(z)$ and $f_{j}(z)$ determine the variations in the displacements through the thickness of the plate; $\phi_{j}=Z_{j}^{(o)} / b$ have been put with $Z_{j}^{(o)}$ the positive zeros of the zero-order $J_{0}(r)$ Bessel function [6]. If the fields $u(r, z)$ and $w(r, z)$ are continuous and of bounded variation in the subinterval $(p, q)$ of $(0, b)$, such expansions are uniformly convergent throughout the interval $(p+\varepsilon, q-\varepsilon)$, with $\varepsilon>0$ [7]. 
It is observed that the requirement $w(b, z)=0$ is verified by the representation form of $w$ for $r=b$.

By using the equilibrium equations, the following expressions for the functions $g_{j}(z)$ and $f_{j}(z)$ are obtained

$$
\begin{aligned}
f_{j}(z)=C_{1}^{(j)} \cosh \left(\phi_{j} z\right)+C_{2}^{(j)} \sinh \left(\phi_{j} z\right)+C_{3}^{(j)} z \cosh \left(\phi_{j} z\right)+C_{4}^{(j)} z \sinh \left(\phi_{j} z\right), \\
g_{j}(z)=-\frac{1}{\phi_{j}}\left(C_{1}^{(j)} \sinh \left(\phi_{j} z\right)+C_{2}^{(j)} \cosh \left(\phi_{j} z\right)\right)-\frac{1}{\phi_{j}^{2}} C_{3}^{(j)}\left(\frac{(\lambda+3 \mu)}{(\lambda+\mu)} \cosh \left(\phi_{j} z\right)+z \phi_{j} \sinh \left(\phi_{j} z\right)\right)+ \\
-\frac{1}{\phi_{j}^{2}} C_{4}^{(j)}\left(\frac{(\lambda+3 \mu)}{(\lambda+\mu)} \sinh \left(\phi_{j} z\right)+z \phi_{j} \cosh \left(\phi_{j} z\right)\right)
\end{aligned}
$$

and the coefficients $C_{1}^{(j)}, C_{2}^{(j)}, C_{3}^{(j)}$ and $C_{4}^{(j)}$ will be uniquely determined by the boundary conditions.

Due to the complexity of the contact problem, traction conditions are introduced on the upper and lower faces of the plate in the form

$$
\begin{aligned}
& \sigma_{r z}(r, \pm h)=0, \\
& \sigma_{z z}(r,-h)=0, \\
& \sigma_{z z}(r,+h)=p(r) .
\end{aligned}
$$

where $p(r)$ is the normal load on the upper face of the plate which is written as the following Fourier Bessel expansion

$$
\begin{gathered}
p(r)=\sum_{j=1}^{\infty} A_{j} J_{0}\left(\phi_{j} r\right), \\
A_{j}=\frac{2 \int_{0}^{a} r p(r) J_{0}\left(\phi_{j} r\right) d r}{b^{2} J_{1}\left(b \phi_{j}\right)^{2}} .
\end{gathered}
$$

A detailed discussion of the expansion convergence can be found in Watson [6].

By assuming the conditions (3) and the form (4) the expressions of the coefficients are obtained

$$
C_{1}^{(j)}=A_{j} \Omega_{1}, \quad C_{2}^{(j)}=A_{j} \Omega_{2}, \quad C_{3}^{(j)}=A_{j} \Omega_{3}, \quad C_{4}^{(j)}=A_{j} \Omega_{4},
$$

where the terms $\Omega_{1}, \Omega_{2}, \Omega_{3}$ and $\Omega_{4}$ assume the form 


$\Omega_{1}=\frac{e^{\phi_{j} h}\left[(\lambda+\mu) \phi_{j} h\left(e^{2 \phi_{j} h}-1\right)+(2 \mu+\lambda)\left(e^{2 \phi_{j} h}+1\right)\right]}{2 \mu(\lambda+\mu) \phi_{j}\left(e^{4 \phi j h}-4 \phi_{j} h e^{2 \phi j h}-1\right)}$
$\Omega_{2}=\frac{e^{\phi_{j} h}\left[(\lambda+\mu) \phi_{j} h\left(e^{2 \phi_{j} h}+1\right)+(2 \mu+\lambda)\left(e^{2 \phi_{j} h}-1\right)\right]}{2 \mu(\lambda+\mu) \phi_{j}\left(e^{4 \phi_{j} h}+4 \phi_{j} h e^{2 \phi_{j} h}-1\right)}$
$\Omega_{3}=-\frac{e^{\phi_{j} h}\left(e^{2 \phi_{j} h}-1\right)}{2 \mu \phi_{j}\left(e^{4 \phi_{j} h}+4 \phi_{j} h e^{2 \phi_{j} h}-1\right)}$
$\Omega_{4}=-\frac{e^{\phi_{j} h}\left(e^{2 \phi_{j} h}+1\right)}{2 \mu \phi_{j}\left(e^{4 \phi_{j} h}-4 \phi_{j} h e^{2 \phi_{j} h}-1\right)}$

The displacement fields are obtained in explicit form from $(1,2)$, by taking into account the explicit coefficients (4) for detailed pre-assigned pressure form $p(r)$ and the coefficient expressions (5).

The contact law is obtained by using the explicit form of the transversal displacement in $z=-h$ and $r=0$ and can be written in the following compact form

$$
\delta=\sum_{j=1}^{\infty} K_{j} A_{j}
$$

where the pressure distribution form allows one to determine the terms of $A_{j}$ by expression (4) and the terms $K_{j}$, taking into account the conditions on the upper and lower face of the plate and the thickness of the plate, assume the explicit following form

$$
K_{j}=\frac{8\left(1-e^{8 \phi_{j} h}-8 \phi_{j} h e^{4 \phi_{j} h}\right)}{\pi R b^{2} \phi_{j}^{4}\left[J_{1}\left(b \phi_{j}\right)\right]^{2}\left(-1+e^{4 \phi_{j} h}+4 \phi_{j} h e^{2 \phi_{j} h}\right)\left(1-e^{4 \phi_{j} h}+4 \phi_{j} h e^{2 \phi_{j} h}\right)} .
$$

Now, we consider the action of a rigid sphere of radius $R$ on a plate in which the contact area radius $a$ of the sphere is small in comparison with the thickness of the plate; in this case, the following normal periodic Hertzian pressure $p(r)$ is assumed acting on the upper face of the plate

$$
p(r)=-\frac{3 P}{2 \pi a^{2}}\left(1-\frac{r^{2}}{a^{2}}\right)^{\frac{1}{2}}, \quad a^{3}=\frac{3 R(2 \mu+\lambda)}{16 \mu(\mu+\lambda)} P,
$$


where $P$ is the resultant of the pressure on the contact area.

The coefficients $A_{j}$ of (4), with the pressure expression (7), assume the form

$$
A_{j}=\frac{16 \mu(\mu+\lambda) \sin \left(a \phi_{j}\right)-a \phi_{j} \cos \left(a \phi_{j}\right)}{\pi b^{2} R(2 \mu+\lambda) \phi_{j}^{3}\left[J_{1}\left(b \phi_{j}\right)\right]^{2}} .
$$

It is remarked that if the contact area of the sphere on the plate is large with respect to the thickness of the plate, the pressure distribution deviates significantly from the Hertzian prediction and a different expression for the pressure distribution has to be assumed $[4,5]$.

\section{Equation of motion}

In this section the problem of the frictionless normal low velocity impact of a rigid sphere against an elastic plate-like body is studied.

By assuming that the period of duration of the impact $\left(2 t_{c}\right)$ is much larger than the time employed by the elastic waves in traversing the plate after the first impact, the contact law presented in the preview section is adopted, in which an explicit form of the contact law is obtained in a static elastic framework taking into account the thickness of the plate $[2,3]$.

The equation of motion during the impact assumes the form

$$
m \ddot{\delta}(t)+P(t)=0 \quad t>0
$$

where $m$ is the mass of the sphere and $P(t)$ the instantaneous resultant pressure.

The contact law obtained in section 2 for the Hertzian pressure distribution (7) may be inverted for $0<\delta<b^{2} / R$ and written as follows [8]:

$$
P=\sum_{k=2}^{\infty} \xi_{k} \delta^{\frac{2 k-1}{3}}
$$

where the first terms of the coefficients $\xi_{k}$ of the inverse expansion are

$$
\xi_{2}=\frac{1}{\gamma_{2}}, \quad \xi_{3}=-\frac{\gamma_{3}}{\gamma_{2}^{\frac{8}{3}}}, \ldots . \text { with } \gamma_{k}=(-1)^{k} \frac{(2 k-2)}{(2 k-1) !}\left(\frac{a}{P^{\frac{1}{3}}}\right)^{2 k-1} \sum_{j=1}^{\infty} \phi_{j}^{2 k-1} K_{j}
$$

The equation of the motion assumes the form

$$
\ddot{\delta}(t)+\frac{1}{m} \sum_{k=2}^{\infty} \xi_{k} \delta(t)^{\frac{2 k-1}{3}}=0
$$

and the initial conditions are 
70 Structures Under Shock and Impact IX

$$
\delta(0)=0 \quad \text { and } \quad \dot{\delta}(0)=v_{0},
$$

where $v_{o}$ is the initial velocity of the sphere before the impact.

The equation (11) may be reduced to the following form

$$
\dot{\delta}(t)=\sqrt{v_{0}^{2}-\frac{3}{m} \sum_{k=2}^{\infty} \frac{\xi_{k}}{k+1} \delta(t)^{\frac{2(k+1)}{3}}} .
$$

and the solution obtained by using a perturbative technique.

The linear term is denoted with $\delta_{o}(t)$ and the first corrective term with $\eta(t)$; in such way, we have

$$
\delta(t)=\delta_{0}(t)+\eta(t)
$$

where

$$
\delta_{0}(t)=v_{0} \sqrt{\frac{m}{\xi_{2}}} \sin \left(\sqrt{\frac{\xi_{2}}{m}} t\right)
$$

It is possible to show that the corrective term may assume the form [1]

$$
\eta(t)=\left(-\int G(t) e^{\int F(t) d t} d t+C\right) e^{-\int F(t) d t}
$$

with the constant $C$ obtained by the condition $\eta(0)=0$; the functions $G(t)$ and $F(t)$ have the following expressions

$$
F(t)=\frac{\delta_{0}(t)\left(\xi_{2}+\xi_{3} \delta_{0}(t)^{\frac{2}{3}}\right)}{m\left(v_{0}^{2}-\frac{\xi_{2}}{m} \delta_{0}(t)^{2}-\frac{3 \xi_{3}}{4 m} \delta_{0}(t)^{\frac{8}{3}}\right)^{\frac{1}{2}}},
$$

and

$$
G(t)=\left(v_{0}^{2}-\frac{\xi_{2}}{m} \delta_{0}(t)^{2}\right)^{\frac{1}{2}}-\left(v_{0}^{2}-\frac{\xi_{2}}{m} \delta_{0}(t)^{2}-\frac{3 \xi_{3}}{4 m} \delta_{0}(t)^{\frac{8}{3}}\right)^{\frac{1}{2}} .
$$

The numerical solution of the equation (13) carries out the indentation, the contact period and the maximum contact force. 


\section{Numerical results and conclusions}

In preview sections low velocity impact against a flexible structure is studied and, in comparison with the impact between compact bodies, an explicit solution taking into account both the dynamic deformation of the structure and the local deformation of the contact region has been obtained.

In this section numerical solutions of the equation (11) are found for a rigid sphere of radius $R=0.13 \mathrm{~m}$ with initial velocity $v_{0}=1 \mathrm{~m} / \mathrm{s}$ in collision against a flexible plate with elastic properties $\lambda=0.36 \mathrm{GPa}$ and $\mu=0.43 \mathrm{GPa}$ and ratio $h / b=0.10$.

By using the perturbative technique introduced in section 3, in fig. 1 the collision history and the influence of the first correction term (16) of the linear term (15) in the equation of motion solution (14) are shown.

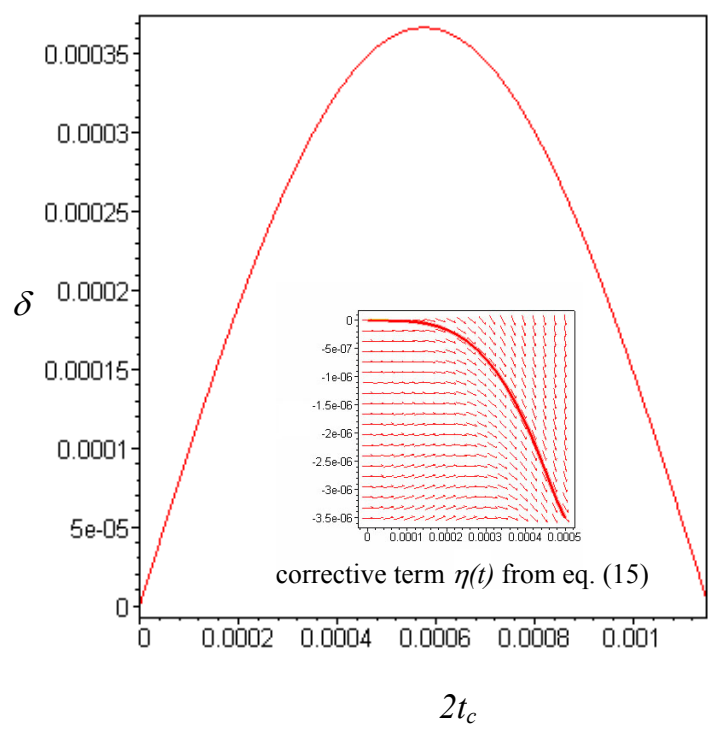

Figure 1: $\quad$ Collision time for $h / b=0.10$.

In fig. 2 it is shown how the time of collision $\left(2 t_{c}\right)$ decreases with the increasing of the ratio $h / b$.

In comparison with Hertz's classical theory it is observed that the deformability of the plate increases the maximum indentation, prolongs the contact period and transfers significant energy into structural vibrations [2].

It is remarked that the mathematical approach utilized to find the contact law in section 2 is very powerful and has been utilized also in [9] to find the indentation in a film/substrate system. 




Figure 2: $\quad$ Time of collision for different ratio $h / b$.

\section{Acknowledgements}

This paper was supported by MIUR Cofin 2004: "Problemi e modelli microstrutturali: applicazioni in ingegneria strutturale e civile" and MIUR Cofin 2005: "Interfacial resistance and failure in materials and structural systems".

\section{References}

[1] Sburlati R., An exact solution for the impact law in thick elastic plates, International Journal of Solids and Structures, Vol.41, pp.2539-2550, 2004.

[2] Stronge W.J., Impact Mechanics, Cambridge University Press, 2000.

[3] Villaggio P., The rebound of an elastic sphere against a rigid wall, Journal of Applied Mechanics, Vol. 63, pp.259-263, 1996.

[4] Keer L.M., Miller G.R., Contact between an elastically supported circular plate and a rigid indenter, International Journal of Engineering Science, Vol.21, pp.681-690, 1983.

[5] Chen C.F., Frederick D., Contact of isotropic square plates with rigid spherical indenters, International Journal of Solids and Structures, Vol.30, pp.637-650, 1993.

[6] Watson G.N., A Treatise on the Theory of Bessel Functions, Cambridge University Press, 1944.

[7] Sneddon I.N., Mixed Boundary Value Problems in Potential Theory, John Wiley \&Sons Inc., 1966.

[8] Morse P.M., Feshbach H., Methods of Theoretical Physics, MacGraw-Hill Press, 1966.

[9] Sburlati, R., Elastic indentation problems in thin films on substrate systems, to appear on Journal of Mechanics of Materials and Structures. 